CDD: 160

\title{
THE ROLE OF CONSISTENCY IN EMPIRICAL SCIENCE
}

\author{
JESÚS MOSTERÍN \\ Académie Internationale de Philosophie des Sciences \\ BELGIQUE \\ Consejo Superior de Investigaciones Cientificas \\ SPAIN
}

jesus@mosterin.com

\begin{abstract}
Any inconsistent theory whose underlying logic is classical encompasses all the sentences of its own language. As it denies everything it asserts, it is useless for explaining or predicting anything. Nevertheless, paraconsistent logic has shown that it is possible to live with contradictions and still avoid the collapse of the theory. The main point of this paper is to show that even if it is formally possible to isolate the contradictions and to live with them, this cohabitation is neither desired by working scientists not desirable for the progress of science. Several cases from the recent history of physics and cosmology are analyzed.
\end{abstract}

Keywords: Consistency. Cosmology. Contradiction. Logic. Physics. Relativity.

\section{CONTRADICTION IN CLASSICAL LOGIC}

In classical logic, a contradiction implies anything. Let $\varphi$ be any sentence. The semantic definition of the truth of a negation $\neg \varphi$ is:

$\neg \varphi$ is true (in interpretation $\mathfrak{I}$ ) if and only if

$\varphi$ is not true (in interpretation $\mathfrak{I}$ )

It follows that under no interpretation can both $\varphi$ and $\neg \varphi$ be true. No interpretation can make $(\varphi \wedge \neg \varphi)$ true. For any formula $\psi$ and any interpretation $\mathfrak{I}$ : if $\mathfrak{I}$ makes $(\varphi \wedge \neg \varphi)$ true, then it also makes $\psi$ true. So, for any formula $\psi:(\varphi \wedge \neg \varphi) \models \psi$. 
Syntactically, from a contradiction (let's say, $\varphi \wedge \neg \varphi$ ) we can infer anything $(\psi)$ in the classical logical calculus: $(\varphi \wedge \neg \varphi) \vdash \psi$.

$$
\begin{array}{rr}
\varphi \wedge \neg \varphi & \\
\varphi & 1) \\
\neg \varphi & 1) \\
\varphi \vee \psi & 2) \\
\psi & \text { 4) and 3) }
\end{array}
$$

\section{THE BANE OF INCONSISTENCY}

From the point of view of classical logic, an inconsistent formal theory (a theory that contains contradictory theorems) is identical to its language. It asserts everything and it denies everything, and so it is utterly useless. To be inconsistent is the worst bane that can afflict a theory, much worse than being false. Small wonder that scientists tend to drop a theory as soon as they discover contradictions in it. If there is no replacement in sight, a period of crisis and uneasiness ensues until a new and hopefully consistent theory is found, which is received by the community with a sigh of relief.

\section{INCONSISTENT THEORIES}

A theory is a set of sentences closed under the relation of consequence $(\models)$. The sentences of a theory are its theorems. Let $\Sigma$ be a set of sentences of language $L: \Sigma \subseteq L$.

$\Sigma$ is a theory

if and only if for every sentence $\alpha \in L$ : if $\Sigma \models \alpha$, then $\alpha \in \Sigma$ if and only if $\Sigma=\{\alpha \in L: \Sigma \models \alpha\}$. 
A theory $T$ is inconsistent

if and only if $T$ is not consistent

if and only if for some $\varphi \in L, \varphi \in T$ and $\neg \varphi \in T$

if and only if $T$ is identical to its language: $T=L(T)$.

Two theories $T$ and $\Sigma$ are incompatible if and only if $T \cup \Sigma$ is an inconsistent theory.

\section{INTUITIONISTIC LOGIC}

Intuitionistic logic is weaker than classical logic. Everything that can be deduced in an intuitionistic system can also be deduced classically. Of course, the interpretation is not the same in both cases (it is constructive in the first case, structural in the second).

The system of classical logic is obtained from intuitionistic logic by adding some new axiom, like Excluded middle $(\varphi \vee \neg \varphi)$ or Double negation $(\neg \neg \varphi \rightarrow \varphi)$.

Intuitionists were afraid of inconsistency. In fact, they were much more afraid of contradiction than classical mathematicians were. From that point of view, intuitionism is more strongly opposed to paraconsistent logic than classical logic is.

A great advantage of intuitionistic logic was supposed to be that it was safer than its classical counterpart. But Gödel proved in 1932 that intuitionistic logic is not safer than classical logic. Any classical formula can be translated into an intuitionistic one in such a way that any classical contradiction would immediately produce an intuitionistic contradiction.

\section{PARACONSISTENT LOGIC}

The motivation for paraconsistent logic is the contrary of the intuitionistic preoccupation. Instead of being too afraid of inconsistency, 
paraconsistent logicians are ready to live with contradictions, if they can only find a way of doing so without producing new contradictions. And they have found it. They have built systems of logic that are weak enough for contradictions to be isolated and sanitized.

Paraconsistent logics (PL) are weaker than classical logic (CL); they allow for fewer inferences to be drawn. For any set $\Gamma$ of formulas and any formula $\varphi$ :

$$
\text { If } \Gamma \vdash_{P L} \varphi \text {, then } \Gamma \vdash_{C L} \varphi \text {. PL } \subset \mathrm{CL} \text {. }
$$

Perhaps the first precedent of paraconsistent logic, although driven by quite different motivations, was the logic of strict implication (or strict conditional), presented by Clarence I. Lewis in 1918 as an alternative to the "material" implication he found and disliked in the writings of Bertrand Russell. Lewis defined " $\varphi$ strictly implies $\psi$ " as "it is not possible that both $\varphi$ and not $\psi$ ": $\neg \diamond(\varphi \wedge \neg \psi)$. Of course, in Lewis' logic, it is not the case that a falsehood strictly implies anything. But neither is it the case in classical logic: it is contradiction, not just falsehood that implies anything. Another related precedent of paraconsistent logic is to be found in relevance logic, which requires that the antecedent and consequent of a conditional be relevantly related for the conditional to be true. This relevance relation is usually defined by the syntactical constraint that some words (or some constants, predicates or atomic formulae, in the case of a formalized language) have to be shared by the antecedent and the consequent. Ivan Orlov in 1928 had the first idea of relevance logic; Nuel Belnap and Alan R. Anderson gave it in 1975 and 1992 its most accomplished presentation.

Latin American logicians have played a leading role in the development of paraconsistent logic. Newton da Costa presented in 1963 the first formal system (or hierarchy of formal systems) of paraconsistent propositional logic. In 1966, the Argentinean Florencio G. Asenjo published his Logic of Paradox. In 1976, the Peruvian Francisco Miró 
Quesada coined the expression "paraconsistent logic". The Brazilian disciples of Newton da Costa, including Ayda Arruda, Walter Carnielli and Itala Loffredo d'Ottaviano, have made noteworthy contributions to the study of paraconsistency, and so has Jean-Yves Béziau, born in France but living in Brazil.

Newton da Costa and other logicians have shown us that it is possible to build consistent systems of paraconsistent logic, systems that allow us to isolate the found contradictions and so preserve the inconsistent theory from collapse or "explosion". Paraconsistent logics are weaker than classical logic, they allow for fewer inferences to be drawn. It is this weakness that makes it possible to contain the deleterious effects of contradictions. But here lurks a problem. Many domains of science -from physics to economics- are extensions of underlying mathematical theories, and it is not clear that all the power of the underlying mathematics can be preserved under a paraconsistent reconstruction.

\section{THE REGULATORY IDEA OF CONSISTENCY}

In dealing with inconsistent theories of empirical science, we could replace classical logic with paraconsistent logic. This move would allow us to isolate the found contradictions and so preserve the inconsistent theory from collapse into the whole language. We could continue to use our old theory, even after the red spots of contradiction have appeared on its face. Yes, it is possible, but is it desirable?

The notion of consistency plays the role of a regulatory idea (in the Kantian sense) in the development and progress of science. We almost never know if our theories are consistent, but the discovery of a contradiction always produces a deep crisis and pushes us to make strenuous efforts to find or invent a new and more satisfactory theory.

Let us review some well known cases from the history of physics and cosmology. 


\section{SPECIAL RELATIVITY}

Newton's mechanics reigned as the undisputed foundation of classical physics up to the development of Maxwell's theory of electromagnetism in the middle of the 19th century. At the beginning of the 20th century, physicists became painfully aware of the incompatibility of Maxwell's electrodynamics with Newton's mechanics and with the pre-relativity notions of spacetime structure. A growing uneasiness spread among the members of the community.

In the electromagnetic wave equation (a consequence of Maxwell's equations) neither the velocity of the emitter nor the velocity of the observer appears, as would be required by Newtonian theory. The equations of Newtonian mechanics are invariant under Galilean transformations. Maxwell's equations of electromagnetism are not; instead, they are invariant under Lorentz transformations. The Galilean time transformation (between time coordinates $t$ and $t^{\prime}$ ) is just: $t^{\prime}=t$. The Lorentz time transformation is:

$$
t^{\prime}=\frac{1}{\sqrt{1-\frac{v^{2}}{c^{2}}}}\left(t-\frac{v x}{c^{2}}\right)
$$

When the velocity $v$ approaches 0 , the Lorenz transformation approaches the Galilean transformation, $t^{\prime}=t$. But at high velocities, both formulas diverge dramatically and give completely different values. The perceived inconsistency between Newtonian mechanics and Maxwell's electromagnetism led Albert Einstein to the invention in 1905 of the special theory of relativity. Dissatisfaction with inconsistency was the engine driving the search for a new theory. 


\section{GENERAL RELATIVITY}

Maxwell's equations were compatible with special relativity from the beginning, but Newton's were not. (For example, a particle's mass is constant in Newtonian mechanics, but it is dependent on velocity in special relativity). So, the introduction of special relativity had solved the consistency problem as far as electromagnetism was concerned, but it was impossible to accommodate Newtonian dynamics in the relativistic spacetime framework. The only way out of the contradiction was to drop Newtonian dynamics altogether and to create a new theory of gravitation from scratch: the theory of general relativity.

It was the perceived contradiction between Newtonian gravity and special relativity that led Einstein (and David Hilbert) to search for a new theory of gravity. After several years of agonizing search, in 1915 Einstein found and presented the field equations of general relativity, where gravity was identified with the curvature of spacetime.

In each of these steps, the uneasiness with the inconsistency led to a great theoretical progress that avoided the contradiction, increased the scope of the theory and preserved the previous results (as effective theories in their limited domain of validity). Asymptotically, as velocities become smaller and smaller, the Lorentz transformations converge to the Galileo transformations and the Newtonian results coincide with the special-relativistic. In the same way, asymptotically, as densities become smaller and smaller, the results of general relativity coincide with those of special relativity. So, the well-tested results of a previous theory, for example, the results of Newtonian mechanics in the domain of low velocities (like the ones found on the surface of the Earth) are preserved, even if a contradiction is discovered in the application of that theory outside of its limited domain of validity. But the contradiction is deemed unacceptable by the scientific community and immediately the feverish search for a consistent and more general theory is launched. 


\section{QUANTUM FIELD THEORY}

The exploration of the atomic and subatomic domains opened a new world of amazing and unexpected facts incompatible with classical physics. In order to explain them, a new mechanics had to be invented: quantum mechanics. In 1926, Erwin Schrödinger offered a convenient wave formulation of quantum mechanics based on his just discovered equation. Schrödinger's equation describes the evolution in time of the quantum system. It was soon discovered that Schrödinger's equation is not Lorentz invariant. So, classical quantum mechanics is not compatible with special relativity. That situation could not be tolerated, as special relativity had been thoroughly checked. So, it was the classical formulation of quantum theory that had to yield. A new quantum mechanical theory had to be developed, combining the ideas of quantum mechanics with those of special relativity: Quantum field theory.

It was the perceived inconsistency between quantum mechanics and the special theory of relativity that led Paul Dirac and others to the invention of quantum field theory. In 1928, Dirac formulated a relativistic quantum mechanical wave equation of the electron. Of course, Dirac's equation is Lorentz invariant. Still, the development of quantum field theory was later plagued by the infinities that arose in the computation of several physical magnitudes. This challenge was met in the 1940s by Ichiro Tomonaga, Julian Schwinger and Richard Feynman. In the words of Steven Weinberg, "Quantum field theory is the way it is because $(\ldots)$ it is the only way to reconcile the principles of quantum mechanics with those of special relativity" (cf. Weinberg (1995)). 


\section{SUPERSTRING THEORIES}

Today, the two main pillars of physics are the general theory of relativity and quantum field theory. The big problem looming on the horizon is that both theories are incompatible. The smoothly continuous curvature of spacetime in general relativity contrasts with the frantic, discrete and bumpy quantum world. The problem remains somehow in the background, because the practicing physicists do not use both theories simultaneously. Particle physicists use quantum field theory to explore the very small things that make up the atomic and subatomic world. In this world gravitation is extremely weak and can be safely ignored. For example, the electrostatic force with which the proton in a hydrogen atom attracts the electron is almost $10^{40}$ times stronger than the gravitational attraction between the same two particles. So, in particle physics gravitation and its theory (general relativity) are just ignored; so, no conflict arises. On the other hand, the universe at large, and the huge galaxies and clusters of galaxies and stars and dust clouds that are its most prominent components, are almost exclusively dominated by gravitation. General relativity, our best theory of gravitation, is the basis of the cosmological models. Quantum interactions can be generally ignored. But this separation is sometimes broken, like in the study of black holes, where huge amounts of matter (with the potentiality to distort spacetime according to general relativity) are compressed into a tiny space, where quantum effects are unavoidable. In these exceptional situations, physicists feel quite uncomfortable and suffer under the imminent risk of contradiction.

It is the unhappiness at the incompatibility between the assumptions of general relativity and the standard model of particle physics that is driving the theoretical search of the last twenty years for a theory of quantum gravity that allows for a consistent use of both theories at the same time and on the same problem. The best known candidates are the theories of superstrings (supersymmetric strings). 


\section{AGE OF THE EARTH}

In the second half of the 19th century, under the influence of Charles Lyell and Charles Darwin, many geologists and evolutionary biologists concluded that the Earth had to be billions of years old in order to accommodate the slow processes of geology and evolution. Nevertheless, when the physicists began making calculations, they arrived at much shorter age estimates. William Thomson (Lord Kelvin) was able to calculate how long it would take for a lump of rock the size of the Earth to cool down to its present state from an initial molten state. He estimated for the Earth an age of between 20 and 40 million years. The German physicist Hermann von Helmholtz computed an age of 22 million years; and the Canadian astronomer Simon Newcomb, 18 million years. The blatant contradiction between the widely divergent hypotheses held by naturalists on the one hand and by physicists on the other generated much uneasiness and even acrimony between both communities.

At the end, the geologists and biologists were right and the inconsistency was resolved by the general acceptance of their thesis of an age for the Earth of several billions years (4.54 billion years, according to current estimates). How did the best physicists obtain such off the mark results? Their calculations were correct, but they ignored the effect of radioactivity, which had not yet being discovered. They did not take into account the heat that continued to be generated inside the Earth by the process of radioactive decay. Once radioactivity discovered at the end of the 19th century and its effect added to the calculations, the contradiction was resolved and a deeper understanding of the internal processes of the Earth was achieved. 


\section{HUBBLE CONSTANT AND THE AGE OF THE UNIVERSE}

A similar case arose in the middle of the 20th century concerning the age of the universe (the time elapsed since the big bang). This age is calculated as a function of Hubble's constant $H_{0}$. Till 1958, the measurements of $H_{0}$ were crude and inaccurate. As a consequence, the universe was estimated to be younger than it is. On the other hand, nuclear physics allowed astrophysicists to compute the age of the globular clusters of stars in our galaxy. It turned out that the universe had to be younger that some of its own constituents, like the globular clusters, what is impossible. This contradiction created much anxiety. Finally, it was resolved by new and much more accurate measurements of $H_{0}$. Today, we estimate the age of the universe to be around 13.7 billion years, which corresponds to a value of the Hubble constant of around $74.2 \mathrm{~km} / \mathrm{s} / \mathrm{Mpc}$.

\section{DARK MATTER}

In 1933, Fritz Zwicky was examining the movements and mass distribution of the galaxies in the outskirts of the Coma cluster. When he applied the virial theorem of classical mechanics to the observed movements, Zwicky was surprised to find that the amount of mass implied had to be much larger than the aggregated mass of the stars and other luminous material. These results were in contradiction with the tacit assumption that most of the matter in the universe is luminous, matter emitting light or other electromagnetic radiation, as is the case with the stars and galaxies we see in the night sky. This is the "problem of the missing mass". Zwicky could not accept that contradiction. In 1934 he dropped the assumption that most matter is luminous. Instead, he postulated a new type of matter that does not enter into electromagnetic interactions, but has gravitational effects. This he called dark matter.

Manuscrito - Rev. Int. Fil., Campinas, v. 34, n. 1, p. 293-305, jan.-jun. 2011. 
At that time, most astronomers were not aware of Zwicky's investigations. Some forty years later, new studies of motions of stars within galaxies also implied the presence of a large halo of unseen matter extending beyond the visible disk of their galaxy; other studies of the orbital movements of galaxies in their clusters also led to the same conclusion. Suddenly, most astronomers became aware of the inconsistency. Zwicky's dark matter proposal was later confirmed by studies of gravitational lensing and cosmological expansion rates. Nowadays, dark matter has been fully incorporated into the standard cosmological model. According to current estimations, there is five times more dark matter than ordinary matter.

\section{ACCELERATION OF THE EXPANSION AND DARK ENERGY}

We estimate that the combined mass of ordinary matter and dark matter make up some $27 \%$ of the energy of the universe. The rest, $73 \%$, is supposed to exist in the form of dark energy. We do not know what dark energy is; actually, "dark energy" is just the name we give to our ignorance concerning what causes the acceleration of the observed expansion of the universe.

Up to 1998, almost everyone in the cosmological community believed that the expansion of the universe is slowing down due to the pull of gravitation, which acts like a brake. So, there was general amazement at the discovery that the expansion of the universe is actually accelerating, and not decelerating, as previously assumed. This conclusion followed from new and coincident measurements of the distances to far away type $I_{a}$ supernovae independently made by the teams of Saul Perlmutter in California and Brian Schmidt in Australia. These results contradicted the previous assumptions of the standard cosmological model. In order to avoid the inconsistency, those assumptions 
were dropped and a universe model with $73 \%$ of dark energy was introduced. This is not very satisfying, as no one knows what we are talking about. But even this desperate resource to an unknown but pervasive"dark energy" was deemed preferable to just remaining one day longer in the contradiction.

\section{CONCLUSION}

There are technological domains, like programming and software development, where bugs are being detected and corrected all the time. They are very different from pure mathematics, defined by Cantor as the kingdom of freedom, where you can do whatever you want, as long as you do not contradict yourself. And they are very different from empirical science. As a matter of fact, they are not (and they do not pretend to be) a science. But in all of science, and especially in empirical science, the regulatory idea of consistency plays a crucial role in the dynamics of scientific progress.

The discovery of a contradiction in empirical science is experienced as a trauma. It produces uneasiness, discomfort and anxiety across the community involved. Caught in this type of conundrum, it is not a way of living with contradiction that scientists are looking for, but a way of jumping out of contradiction. And the few scientists that find a way of restoring consistency through strenuous effort and sheer creativity are justly hailed as intellectual heroes by the rest of the community.

\section{REFERENCES}

WEINBERG, S. The Quantum Theory of Fields, vol. I, p. XXI. Cambridge University Press, 1995. 